\title{
Methylguanine methyltransferase- mediated in vivo selection and chemoprotection of allogeneic stem cells in a large-animal model
}

\author{
Tobias Neff, ${ }^{1}$ Peter A. Horn, ${ }^{1}$ Laura J. Peterson, ${ }^{1}$ Bobbie M. Thomasson, ${ }^{1}$ \\ Jesse Thompson, ${ }^{1}$ David A. Williams, ${ }^{2}$ Manfred Schmidt,${ }^{3}$ George E. Georges, ${ }^{1,4}$ \\ Christof von Kalle, ${ }^{2}$ and Hans-Peter Kiem ${ }^{1,4}$
}

${ }^{1}$ Clinical Research Division, Fred Hutchinson Cancer Research Center, Seattle, Washington, USA

${ }^{2}$ Division of Experimental Hematology, Children's Research Foundation, Cincinnati Children's Hospital Medical Center, Cincinnati, Ohio, USA

${ }^{3}$ Institute for Molecular Medicine and Cell Research, University of Freiburg, Freiburg, Germany

${ }^{4}$ Department of Medicine, University of Washington, Seattle, Washington, USA

\begin{abstract}
Clinical application of gene therapy for genetic and malignant diseases has been limited by inefficient stem cell gene transfer. Here we studied in a clinically relevant canine model whether genetic chemoprotection mediated by a mutant of the DNA-repair enzyme methylguanine methyltransferase could circumvent this limitation. We hypothesized that genetic chemoprotection might also be used to enhance allogeneic stem cell transplantation, and thus we evaluated methylguanine methyltransferase-mediated chemoprotection in an allogeneic setting. We demonstrate that gene-modified allogeneic canine $\mathrm{CD}_{34}{ }^{+}$cells can engraft even after low-dose total body irradiation conditioning. We also show that cytotoxic drug treatment produced a significant and sustained multilineage increase in gene-modified repopulating cells. Marking in granulocytes rose to levels of up to $98 \%$, the highest in vivo marking reported to date to our knowledge in any large-animal or human study. Increases in transgene-expressing cells after in vivo selection provided protection from chemotherapy-induced myelosuppression, and proviral integration site analysis demonstrated the protection of multiple repopulating clones. Drug treatment also resulted in an increase in donor chimerism. These data demonstrate that durable, therapeutically relevant in vivo selection and chemoprotection of genemodified cells can be achieved in a large-animal model and suggest that chemoprotection can also be used to enhance allogeneic stem cell transplantation.
\end{abstract}

J. Clin. Invest. 112:1581-1588 (2003). doi:10.1172/JCI200318782.

\section{Introduction}

The therapeutic potential of hematopoietic stem cell gene therapy has been realized only recently with the

Received for publication April 29, 2003, and accepted in revised form September 9, 2003.

Address correspondence to: Hans-Peter Kiem, Fred Hutchinson Cancer Research Center, Clinical Research Division, D1-100, 1100 Fairview Avenue N, PO Box 19024, Seattle, Washington 98109-1024, USA. Phone: (206) 667-4425; Fax: (206) 667-6124; E-mail: hkiem@fhcrc.org.

Peter A. Horn's present address is: Institute for Transplantation Diagnostics and Cell Therapeutics, Heinrich-Heine-University, Düsseldorf, Germany.

Tobias Neff and Peter A. Horn contributed equally to this work.

Conflict of interest: The authors have declared that no conflict of interest exists.

Nonstandard abbreviations used: $\mathrm{O}^{6}$-methylguanine-DNA methyltransferase (MGMT); 1,3-bis-(2-chloroethyl)-1nitrosourea (BCNU); $\mathrm{O}^{6}$-benzylguanine $\left(\mathrm{O}^{6} \mathrm{BG}\right)$; dog leukocyte antigen (DLA); stem cell factor (SCF); total body irradiation (TBI); P140K mutant of MGMT (MGMT[P140K]); viruscontaining medium (VCM); internal ribosomal entry site (IRES); enhanced green fluorescent protein (EGFP); spleen

focus-forming virus (SFFV); variable number of tandem repeat (VNTR); linear amplification-mediated PCR (LAM-PCR); murine stem cell virus (MSCV). successful treatment of patients with SCID (1-3). A critical limitation to stem cell gene therapy has been the low gene-transfer efficiency with available vectors in clinical trials or clinically relevant large-animal models, and more recently the risk of insertional mutagenesis (4-6). Over the last few years, advances such as gene transfer on fibronectin fragment $\mathrm{CH}-296$ (RetroNectin; generously provided by Takara Bio Inc., Otsu, Japan) (7, 8), improved growth factor combinations (8-10), novel pseudotypes $(8,11-13)$, and the development of packaging cell lines based on human rather than rodent cell lines (14) have all contributed to advance stem cell gene therapy to the threshold of clinical utility. However, despite this progress, gene transfer is still variable and not consistently in the therapeutic range. Furthermore, the most encouraging studies required transplantation of genetically modified cells after myeloablative conditioning, whereas gene-marking levels (proportions of genetically modified circulating blood cells) after moredesirable reduced-intensity conditioning regimens have been substantially lower (15).

In vivo selection has been proposed as a strategy to increase the level of in vivo gene marking by conferring 
a selective survival advantage to the transduced cell population. Proof of principle for this strategy has been provided recently by clinical trials of patients with SCID (1-3), in which therapeutic transgenes (encoding either the common $\gamma$-receptor chain or adenosine deaminase) compensated for the intrinsic survival disadvantage of diseased lymphocytes and their precursors, thus allowing for the reconstitution of a functional lymphoid system from a small number of genetically corrected stem or progenitor cells. For most diseases, the therapeutic transgene does not confer a sufficient survival advantage. Treatment of these diseases could therefore be improved by the incorporation of a selectable marker gene such as a growth-switch gene $(16,17)$ or a drugresistance gene (18) in addition to the therapeutic gene to allow for in vivo selection.

One of the most promising drug-resistance genes is $O^{6}$-methylguanine-DNA methyltransferase (MGMT), which encodes a DNA-repair enzyme that can confer resistance to the cytotoxic effects of nitrosoureas such as 1,3-bis-(2 chloroethyl)-1-nitrosourea (BCNU) and to methylating agents such as temozolomide. $\mathrm{BCNU}$ produces profound hematopoietic stem cell toxicity, a prerequisite for successful stem cell selection. Therefore, this selection system could allow for both in vivo selection and marrow protection in dose-intensified cancer chemotherapy regimens. To accentuate hematopoietic toxicity, endogenous activity of MGMT in stem cells (or tumor cells) can be inactivated by $O^{6}$-benzylguanine $\left(\mathrm{O}^{6} \mathrm{BG}\right)$. In contrast, the mutant forms of MGMT used for stem cell protection are insensitive to the inhibitory activity of ${ }^{6}{ }^{6} \mathrm{BG}$. Successful MGMT-mediated in vivo selection of murine stem cells using $\mathrm{O}^{6} \mathrm{BG}$ and either $\mathrm{BCNU}$ or temozolomide has been described (19-21). However, stem cell gene-transfer studies in the mouse model have generally not been predictive of outcomes in large animals or in humans.

Here, we studied whether a combination of $\mathrm{O}^{6} \mathrm{BG}$ and BCNU can select MGMT-transduced stem cells in the dog. We chose the canine model because it is a well-established preclinical model for testing both gene therapy (13) and allogeneic transplantation strategies (22). To provide proof of principle for the hypothesis that in vivo selection could be used in the setting of allogeneic stem cell transplantation, we studied the MGMT selection system after transplantation of gene-modified dog leukocyte antigen-matched (DLA-matched) allogeneic CD34 cells, the canine equivalent of a human leukocyte antigen-matched (HLA-matched) sibling graft in humans.

\section{Methods}

Animals. Dogs were raised and housed at the Fred Hutchinson Cancer Research Center under conditions approved by the American Association for Accreditation of Laboratory Animal Care (10). Animal experiments were reviewed and approved by the Fred Hutchinson Cancer Research Institutional Animal Care and Use Committee. Marrow was obtained from dogs sedated by general anesthesia. DLA-identical littermates were selected for transplantation based on identity for highly polymorphic MHC class I and class II microsatellite markers and identity for DLA DRB1 alleles as determined by direct sequencing $(23,24)$. DLA-matched littermate donors were treated with canine stem cell factor (SCF) $(25 \mu \mathrm{g} / \mathrm{kg}$ body weight subcutaneously, once daily) and canine G-CSF ( $5 \mu \mathrm{g} / \mathrm{kg}$ body weight subcutaneously, twice daily) for 5 consecutive days before $\mathrm{CD} 34^{+}$cells were isolated from bone marrow or by leukapheresis (Table 1) using established methods (25). In preparation for transplantation, the animals received a single dose of either $920 \mathrm{cGy}$ or $400 \mathrm{cGy}$ total body irradiation (TBI). Post-transplantation immunosuppression consisted of cyclosporine ( $15 \mathrm{mg} / \mathrm{kg}$ body weight orally, twice daily). Animals treated with low-dose irradiation (400 cGy) also received mycophenolate mofetil $(10 \mathrm{mg} / \mathrm{kg}$ body weight subcutaneously, twice daily) (24).

Viral vectors. The oncoretroviral vector plasmid MIEG3P140K was generated by cloning the cDNA encoding the P140K mutant of methylguanine methyltransferase (MGMT[P140K]) into the MIEG3 vector (26). The vector was used to transiently transfect Phoenix-GALV packaging cells (14). The resulting viruscontaining medium (VCM) was used to transduce 293T-based Phoenix-RD114 packaging cells. Briefly, Phoenix-RD114 packaging cells were generated by stable transfection of Phoenix-gp cells (kindly provided by Gary Nolan, Stanford University, Stanford, California, USA) with an expression plasmid for the RD114 envelope protein. A helper virus-free high-titer clone was selected. Retroviral supernatant was collected in DMEM supplemented with $20 \%$ FBS and $1 \%$ penicillin/streptomycin from subconfluent monolayers of 293T-derived clonal Phoenix-RD114 producer cells after incubation for 12 hours at $37^{\circ} \mathrm{C}$. Viral particles were concentrated by centrifugation at $7,277 \mathrm{~g}$ at $4^{\circ} \mathrm{C}$ for 24 hours and resuspension of the pellet in $1 \%$ of the original volume. Recovery of viral particles after centrifugation was typically around $60-80 \%$. The viral producer clone was tested for production of replication-competent helper virus and was found to be negative. Titers were typically $1 \times 10^{7}$ to $2 \times 10^{7}$ infectious particles per milliliter for concentrated Phoenix-RD114 retroviral vector stock.

The lentiviral vector pRRL-cPPT-SFFV-P140K-IRESEGFP.SIN contains a self-inactivating long terminal repeat, the 118-bp polypurine tract (27), and directs expression of a transcript encoding MGMT(P140K), an internal ribosomal entry site (IRES), and enhanced green fluorescent protein (EGFP) from an internal spleen focus-forming virus (SFFV) promoter. Lentiviral vector was produced and concentrated using established methods (28). Titers were $2 \times 10^{8}$ to $4 \times 10^{8}$ infectious particles per milliliter for concentrated lentiviral vector stocks.

Transduction of $\mathrm{CD} 34^{+}$cells. Retroviral transductions of $\mathrm{CD} 34^{+}$cells were carried out on a RetroNectin-coated surface. After 48 hours of prestimulation with canine G-CSF, canine SCF, human Flt3-ligand, and human thrombopoietin (all at $50 \mathrm{ng} / \mathrm{ml}$ ), cells were exposed for 4 hours to a dilution of concentrated vector at a MOI of 3-4 in the presence of growth factors. After 
Table 1

Engraftment of gene-modified allogeneic stem cells

\begin{tabular}{|c|c|c|c|c|c|c|}
\hline Animal & $\begin{array}{l}\text { Stem cell } \\
\text { source }\end{array}$ & $\begin{array}{l}\text { Number of } \\
\text { CD34-selected } \\
\text { transduced } \\
\text { cells infused }\end{array}$ & $\begin{array}{l}\% \text { FACS } \\
\text { on day } 6\end{array}$ & $\begin{array}{l}\text { Time to } \\
\text { granulocyte } \\
\text { recovery }\end{array}$ & $\begin{array}{l}\text { Time to } \\
\text { platelet } \\
\text { recovery }\end{array}$ & $\begin{array}{l}\text { Follow-up } \\
\text { (weeks) }\end{array}$ \\
\hline G069 & Allo BM & $92 \times 10^{6} / \mathrm{kg}$ & 83 & 14 & 72 & 74 \\
\hline G154 & Allo BM & $39 \times 10^{6} / \mathrm{kg}$ & 66 & 11 & 62 & 53 \\
\hline G250 & Allo PBSCs & $4 \times 10^{6} / \mathrm{kg}$ & 23 & 13 & 39 & 16 \\
\hline G258 & Allo PBSCs & $4 \times 10^{6} / \mathrm{kg}$ & 14 & 14 & 36 & 10 \\
\hline
\end{tabular}

Allo, allogeneic; BM, bone marrow primed in vivo with canine G-CSF and canine SCF; PBSCs, peripheral blood stem cells mobilized with canine G-CSF and canine SCF; FACS, fluorescence-activated cell sorting.

overnight culture in media containing growth factors, cells were re-exposed to the same MOI of concentrated vector for 4 hours. Immediately after this second exposure to VCM (after about 72 hours of total time in culture), cells were washed and reinfused into the irradiated littermate recipient.

Lentiviral transductions were carried out on a RetroNectin-coated surface. Cells were exposed for 18 hours to a dilution of concentrated vector (MOI of 100) in the presence of growth factors canine G-CSF, canine SCF, human Flt3-ligand, and human megakaryocyte growth and development factor (all used at $50 \mathrm{ng} / \mathrm{ml}$ ). After exposure to VCM in the presence of growth factors, cells were washed with PBS and reinfused into the irradiated littermate recipient.

$O^{6} B G / B C N U .50 \mathrm{mg}$ of $\mathrm{O}^{6} \mathrm{BG}$ (Sigma Aldrich, St. Louis, Missouri, USA) were dissolved in $30 \mathrm{ml}$ of $40 \%$ polyethylene glycol in PBS, and the concentration was adjusted to $1 \mathrm{mg} / \mathrm{kg}$ with pre-warmed $\left(37^{\circ} \mathrm{C}\right) \mathrm{PBS}$. The drug was further diluted in normal saline to a final volume of $250 \mathrm{ml}$ and was infused over 15-20 minutes. BCNU (Bristol-Myers Squibb Co., Princeton, New Jersey, USA) was diluted according to the manufacturer's instructions and was further diluted in normal saline to a total volume of $20 \mathrm{ml}$. One hour after the end of $\mathrm{O}^{6} \mathrm{BG}$ infusion, $\mathrm{BCNU}$ was injected over 3-5 minutes.

Flow cytometry. Flow-cytometric quantification of at least 20,000 events (gated by forward and right-angle light scatter and excluded for propidium iodide, 1 $\mu \mathrm{g} / \mathrm{ml}$ ) was performed on a FACSCalibur (BD Biosciences Immunocytometry Systems, San Jose, California, USA). Analysis of flow-cytometric data was performed with CellQuest v3.3 software (BD Biosciences, San Jose, California, USA) with gating to exclude fewer than $0.1 \%$ control cells in the relevant region for white blood cells. Control cells from a normal dog, matching the cell type assayed, were used to set the gates. FloJo software (Tree Star Inc., San Carlos, California, USA) was used to assess the percentage of EGFP-expressing erythrocytes and platelets. The Overton subtraction algorithm was used to subtract background.

Variable number of tandem repeats. The donor chimerism status after transplantation was assessed using a PCR analysis that measures the percentage of polymorphic $(\mathrm{GAAA})_{n}$ repeats, as described $(24,29)$.
Briefly, 10-20 pmol of the $5^{\prime}$ primer was labeled with $\left[\gamma^{-32} \mathrm{P}\right]$ ATP using T4 polynucleotide kinase. The final PCR reaction contained the labeled primer, 10-20 pmol of the reverse primer, $10 \% 10 \times$ PCR buffer with $15 \mathrm{mM}$ magnesium chloride, $200 \mu \mathrm{M}$ deoxyribonucleotide mix, $0.1 \mathrm{U} / \mu \mathrm{l}$ of Platinum Taq DNA polymerase, and $50 \mathrm{ng}$ of genomic sample DNA. The samples were amplified and subsequently separated by $5 \%$ polyacrylamide denaturing gel electrophoresis. Digitalized images of the PCR gels were obtained. Imageanalysis software (ImageQuant; Molecular Dynamics, Sunnyvale, California, USA) was used to estimate the proportion of donor- and host-specific bands.

Linear amplification-mediated PCR. Integration-site analysis by linear amplification-mediated PCR (LAMPCR) was performed on canine DNA isolated from either total bone marrow or peripheral blood leukocytes. LAMPCR was performed as described previously $(30,31)$ with 100 ng DNA serving as the template.

Statistical analysis. A paired $t$ test was used to analyze the mean difference in percentage of gene-marked granulocytes before the first and after the last drug administration in dogs G069, G154, and G250.

\section{Results}

Gene-modified cells engraft in allogeneic canine recipients after bigh-dose and low-dose conditioning. We first studied in two dogs (G069 and G154) the ability of oncoretrovirally transduced canine $\mathrm{CD} 34^{+}$cells to engraft in a DLAmatched littermate recipient after high-dose irradiation with $920 \mathrm{cGy}$. This dose usually results in full donor chimerism if an unmanipulated graft is transplanted, but it has not been tested in conjunction with transplantation of oncoretrovirally transduced allogeneic hematopoietic stem cells. We used an RD114-pseudotype murine stem cell virus-based (MSCV-based) bicistronic oncoretroviral vector (MIEG3P140K) expressing MGMT(P140K) and EGFP. Both the CD34-selected transduced donor stem cells and the CD34-depleted non-transduced donor cells were infused into the irradiated recipient. Both animals engrafted rapidly (Table 1). Chimerism analysis in peripheral blood leukocytes before in vivo selection showed $77-80 \%$ donor chimerism in dog G069 and 53-57\% donor chimerism in $\operatorname{dog}$ G154 at 3 months after transplantation. 
We next studied whether allogeneic gene-modified cells can engraft after reduced-intensity conditioning with low-dose TBI (400 cGy). A potential obstacle to achieving this goal is the reduced engraftment potential of hematopoietic stem cells after the extended culture period necessary for efficient oncoretroviral gene transfer (9). Lentiviral vectors allow for efficient gene transfer to canine repopulating cells after a short overnight transduction protocol (our unpublished observations), which presumably preserves the engraftment potential of gene-modified cells. Thus, we used a lentiviral vector to study the transplantation of gene-marked allogeneic stem cells after low-dose TBI conditioning.

Two dogs (G250 and G258) were conditioned with low-dose TBI and received lentivirally transduced DLAidentical littermate peripheral blood $\mathrm{CD}_{3} 4^{+}$cells mobilized with canine G-CSF and canine SCF. The non$\mathrm{CD} 4^{+}$fraction was also reinfused. Both animals engrafted rapidly (Table 1) and showed an initial donor chimerism of greater than $75 \%$ approximately 4 weeks after transplantation. These data demonstrate the successful gene modification and engraftment of allogeneic stem cells in a large-animal model after a myeloablative dose of TBI (920 cGy) and a less toxic, low-dose TBI (400 cGy) conditioning regimen.

Durable in vivo selection of allogeneic MGMT(P140K)-overexpressing hematopoietic stem cells. To ensure that MGMT(P140K) overexpression in canine hematopoietic cells confers resistance to the combination of $\mathrm{O}^{6} \mathrm{BG}$ and $\mathrm{BCNU}$, we studied in two independent experiments whether dog $\mathrm{CD} 34^{+}$cells transduced with the oncoretroviral vector described above had an in vitro survival advantage compared to nontransduced cells. Vector-transduced canine CD34-selected bone marrow cells were first exposed in vitro for 1 hour to $\mathrm{O}^{6} \mathrm{BG}(20$ $\mu \mathrm{M})$, then no BCNU or different concentrations of BCNU were added for an additional hour, and cells were washed and cultured for an additional 10 days. Flow-cytometric analysis on day 10 revealed that cells exposed to $\mathrm{O}^{6} \mathrm{BG}$ only showed gene-marking levels of $22 \%$ in the first and $23 \%$ in the second experiment, whereas cells exposed to the combination of $\mathrm{O}^{6} \mathrm{BG}$ and BCNU showed gene-marking of $79 \%$ in the first and $84 \%$ in the second experiment, demonstrating preferential survival of transduced cells after exposure to $\mathrm{O}^{6} \mathrm{BG}$ and $\mathrm{BCNU}$. Of note, a higher dose of BCNU (50 $\mu \mathrm{M})$ paradoxically led to a less pronounced selection (56\% EGFP-positive cells versus 22\% EGFP-positive with $\mathrm{O}^{6} \mathrm{BG}$ only), associated with a decline in cell numbers over the 10 days after drug exposure. These data indicate that MGMT(P140K) can protect canine $\mathrm{CD} 34^{+}$ cells in vitro against the toxic effects of $\mathrm{O}^{6} \mathrm{BG}$ and $\mathrm{BCNU}$ and suggest that excessive drug concentrations can overcome the protective effect of MGMT(P140K) overexpression. We next focused on an in vivo evaluation of the MGMT(P140K)-based selection system.

To test MGMT(P140K)-mediated in vivo selection, we treated three of the four dogs with $\mathrm{O}^{6} \mathrm{BG}$ and $\mathrm{BCNU}$. In all three dogs, we were able to demonstrate a statistically significant $(P=0.03)$ increase in gene-modified cells after drug treatment. In the first dog (G069), the percentage of EGFP-positive granulocytes early after transplantation was greater than $30 \%$. By day 100, gene-marking levels stabilized at somewhat lower levels. On day 108 after transplantation, we administered $5 \mathrm{mg} / \mathrm{kg}$ of $\mathrm{O}^{6} \mathrm{BG}$, followed by $0.4 \mathrm{mg} / \mathrm{kg}$ of BCNU. Following drug administration, sustained in vivo selection, as measured by flow cytometry, was observed in all peripheral blood lineages, including granulocytes (Figure 1a), lymphocytes (Figure 1a), red blood cells, and platelets (Figure 1b). Substantial selection was also observed in bone marrow-derived CD34+ cells (Figure 1c) and in bone marrow-derived colony-forming cells (data not shown). Quantitative TaqMan PCR of DNA samples derived from peripheral blood white blood cells was used to confirm selection (Figure 1d). Marking peaked at levels greater than $80 \%$ in granulocytes shortly after a first cycle of drug administration. Four weeks after drug administration, marking stabilized at approximately $54-68 \%$ in granulocytes, $25-45 \%$ in red blood cells, and $18-41 \%$ in platelets. Selection in lymphocytes peaked at approximately $20 \%$. To confirm reproducibility of the selection, and to demonstrate that MGMT(P140K) mediates chemoprotection, this dog (G069) was re-treated with identical doses of $\mathrm{O}^{6} \mathrm{BG}$ and BCNU on day 367 after the first drug administration. The marking in granulocytes rose to values of approximately $98 \%$ after this second round of in vivo selection, with a follow-up of 6 weeks after the second drug dose (Figure 1a). Selection also was observed in the other lineages. Although the first drug administration in this dog caused prolonged neutropenia and thrombocytopenia, both the absolute neutrophil count (not shown) and the platelet count (Figure 1e) remained within normal limits after the second drug administration. These data indicate that MGMT(P140K)-transduced cells are able to provide protection of the bone marrow from the toxic effects of the combination of $\mathrm{O}^{6} \mathrm{BG}$ and $\mathrm{BCNU}$.

In the second dog undergoing in vivo selection (G154), we sought to reduce the hematopoietic toxicity associated with the drug selection in dog G069. In this dog we therefore administered two cycles of $\mathrm{O}^{6} \mathrm{BG}$ and BCNU with a $50 \%$-reduced dose of BCNU $(0.2 \mathrm{mg} / \mathrm{kg})$ followed by one cycle of drug selection with a $25 \%$ reduced dose of $\mathrm{BCNU}(0.3 \mathrm{mg} / \mathrm{kg})$, with treatments separated by approximately 4 weeks. In vivo selection was observed after every cycle of drug selection in granulocytes (Figure 2a), red blood cells, and platelets (Figure $2 \mathrm{~b}$ ), and in bone marrow-derived CD34+ cells (Figure 2c). Gene-marking in this animal has been sustained at approximately $60 \%$ in granulocytes with a follow-up of more than 7 months after the last drug administration. A mild decrease in peripheral white blood cell and platelet counts occurred after every cycle of drug administration; however, counts remained within the normal range throughout the treatment (Figure $2 \mathrm{~d}$ ).

To study in vivo selection after a low-dose TBI conditioning regimen, we gave the third $\operatorname{dog}(\mathrm{G} 250)$, the recipient of a lentivirally transduced DLA-matched littermate 

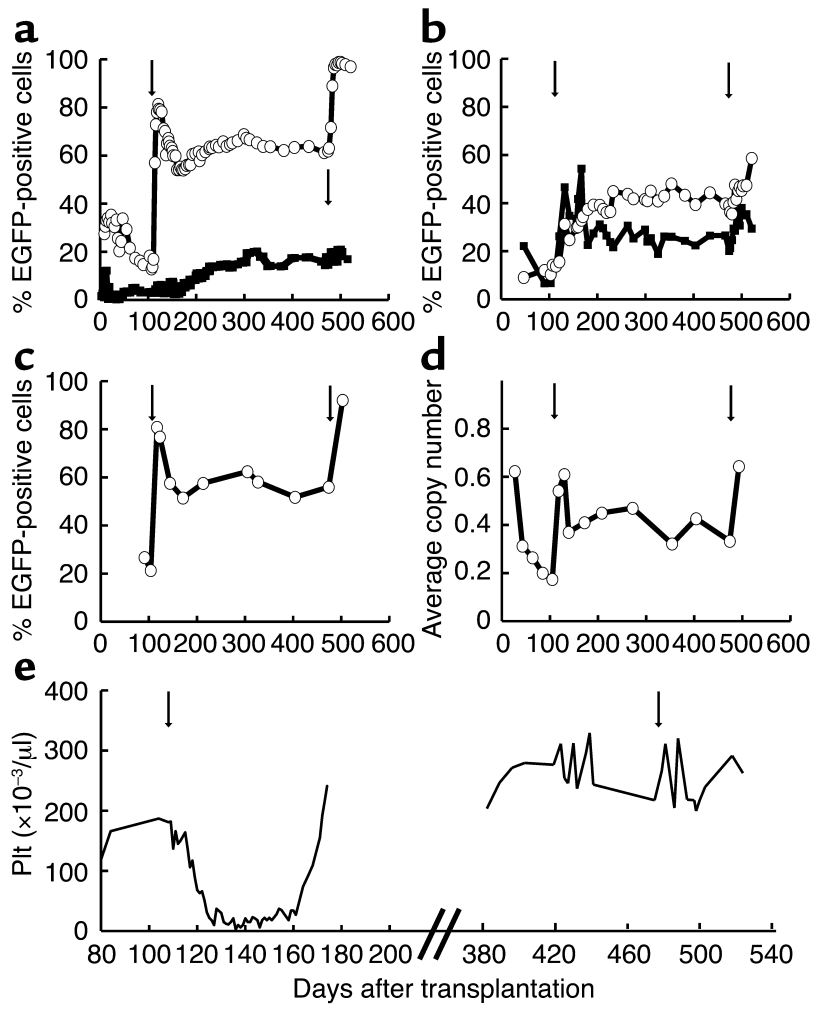

graft, two doses of $\mathrm{O}^{6} \mathrm{BG}$ and $\mathrm{BCNU}(0.3 \mathrm{mg} / \mathrm{kg}$ and 0.4 $\mathrm{mg} / \mathrm{kg}$, separated by 4 weeks). Marking rose from approximately $2 \%$ in granulocytes to a peak of $46 \%$ and stabilized at approximately 20\% (Figure 3a). In vivo selection was also observed in red blood cells and platelets (Figure $3 b)$. These data demonstrate that lentivirally transduced allogeneic stem cells engraft and can be successfully selected in vivo after low-dose TBI conditioning.

Drug-mediated modulation of mixed chimerism. Complete donor chimerism is usually observed after fully myeloablative conditioning and transplantation of unmodified cells. Because the degree of chimerism to be expected after transplantation of cultured, retrovirally transduced cells is not known, we used variable number of tandem repeat (VNTR) analysis $(24,29)$ to study the level of donor chimerism in all animals, including the dogs that received conditioning with $920 \mathrm{cGy}$.

Dog G069 was initially found to have donor contribution from $77 \%$ to $80 \%$. After the first drug administration, donor chimerism was stable for 1 year between $81.5 \%$ and $84.5 \%$. After the second drug administration, there was an increase in donor chimerism to greater than $90 \%$. In the second animal, we observed stable donor chimerism of approximately $55 \%$ in peripheral blood leukocytes and bone marrow before the first drug cycle. After each drug administration, donor contribution increased steadily. After the third drug cycle, donor contribution was greater than 95\% (Figure 4). This level of chimerism was maintained for the entire follow-up period. Importantly, spontaneous conversion to full donor chimerism has not been observed in dogs with stable mixed chimerism at our center (ref. 24 and our unpublished observation).

\section{Figure 1}

In vivo selection in dog G069 after two cycles of $\mathrm{O}^{6} \mathrm{BG}$ and $\mathrm{BCNU}$ separated by 1 year. (a-c) Flow-cytometric analysis. (a) Percent EGFPpositive granulocytes (open circles) and lymphocytes (filled squares) from peripheral blood. (b) Percent EGFP-positive red blood cells (open circles) and platelets (filled squares) from peripheral blood. (c) Percent EGFP-positive CD34+ cells from bone marrow (open circles). (d) Quantification of provirus in leukocytes from peripheral blood by TaqMan PCR (open circles). (e) Platelet counts (PIt) in the time periods following the first and second cycle of $\mathrm{O}^{6} \mathrm{BG}$ and $\mathrm{BCNU}$. The arrows indicate the two cycles of drug administration.

Unexpectedly, both dogs receiving a graft of lentivirally transduced cells after low-dose conditioning showed a high initial degree of donor chimerism $(>75 \%)$ even without in vivo selection. We are currently in the process of defining conditions with further reduced conditioning regimens that will allow for the establishment of stable mixed chimerism in the recipients of lentivirally transduced, DLAmatched littermate grafts.

Protection of multiple repopulating clones. We performed integration-site analyses to characterize the clonal composition of the EGFP-positive compartment before and after the selection process for dogs G069 and G154. LAM-PCR $(30,31)$ of peripheral blood and bone marrow demonstrated that before administration of $\mathrm{O}^{6} \mathrm{BG} /$ $\mathrm{BCNU}$, multiple clones contributed to hematopoiesis (Figure 5). Most of these clones were also detected after selection. We excised 14 sample bands from the gels and determined their sequences. The sequences of four
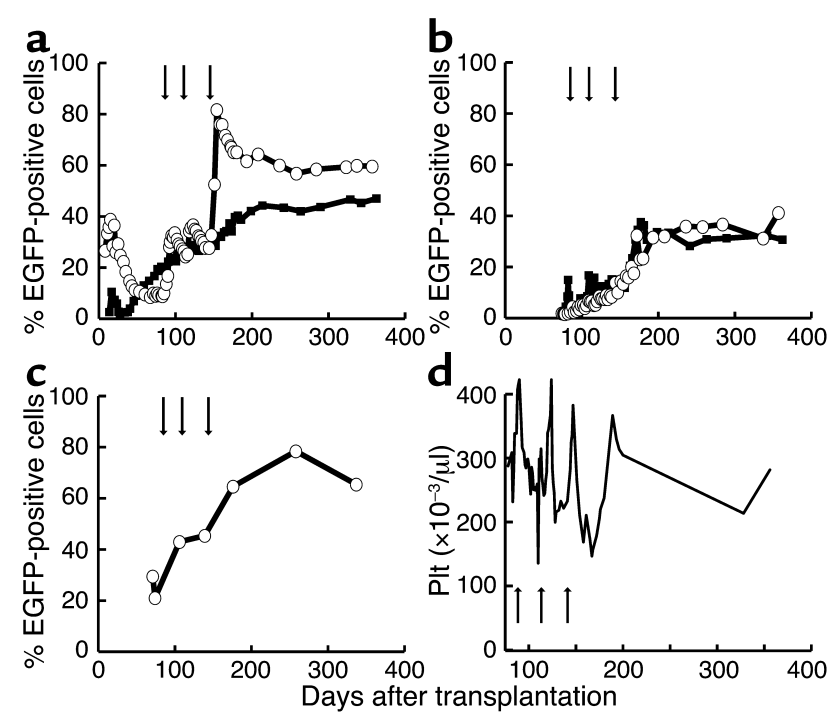

\section{Figure 2}

In vivo selection in dog G154 after three cycles of $\mathrm{O}^{6} \mathrm{BG}$ and $\mathrm{BCNU}$. (a-c) Flow-cytometric analysis. (a) Percent EGFP-positive granulocytes (open circles) and lymphocytes (filled squares) from peripheral blood. (b) Percent EGFP-positive red blood cells (open circles) and platelets (filled squares) from peripheral blood. (c) Percent EGFPpositive $\mathrm{CD} 34^{+}$cells from bone marrow (open circles). (d) Platelet counts in the peripheral blood over time. The arrows indicate the three cycles of drug administration. 


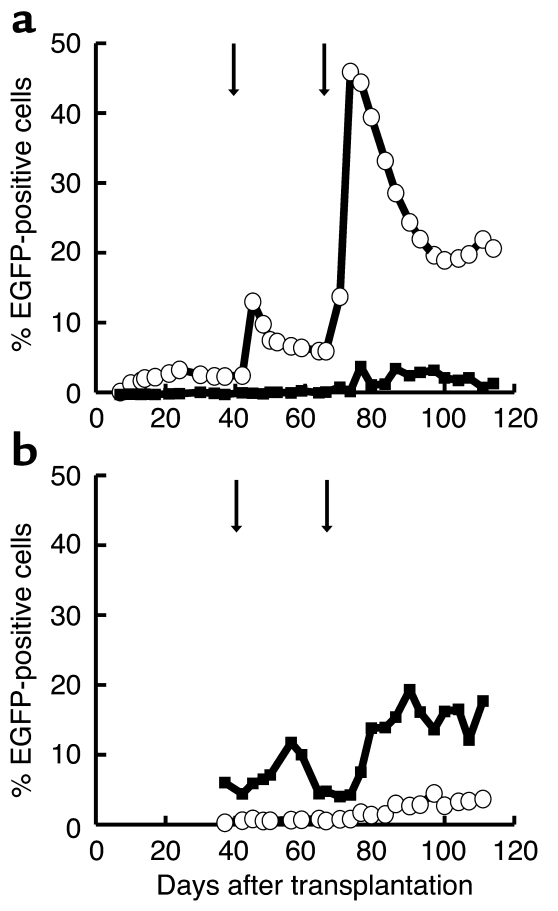

bands were found to have exact matches in bands of the same molecular weight excised at a different time point. These data confirm that bands of identical molecular weight correspond to identical sequences. The data furthermore indicate that in multiple clones the level of MGMT expression was sufficient to allow for protection from the toxic effects of $\mathrm{O}^{6} \mathrm{BG}$ and $\mathrm{BCNU}$. These results confirm our previous finding that a defined number of stem cell clones may be sufficient to support hematopoiesis in large animals (31).

Extrahematopoietic toxicity. The dogs were examined regularly by a board-certified veterinarian and were weighed at least once per week in the 4-week period after drug treatment. Liver function tests and kidney function tests were obtained repeatedly. The only abnormalities noted were increases in liver enzymes and cholestasis parameters in dog G069. These abnormalities were documented before the first cycle of $\mathrm{O}^{6} \mathrm{BG}$ and $\mathrm{BCNU}$ was given and fluctuated over time without apparent correlation to the drug administrations. The etiology of this finding is unclear. All dogs, including G069, have appeared clinically healthy

Figure 4

\section{Figure 3}

In vivo selection in dog G250 after two cycles of $\mathrm{O}^{6} \mathrm{BG}$ and $\mathrm{BCNU}$. Flow-cytometric analysis. (a) Percent EGFP-positive granulocytes (open circles) and lymphocytes (filled squares) from peripheral blood. (b) Percent EGFP-positive red blood cells (open circles) and platelets (filled squares) from peripheral blood. The arrows indicate the two cycles of drug administration.

from the time of neutrophil engraftment until now. All animals maintained their weight within $10 \%$ of baseline after drug administrations. Examination of bone marrow from dogs G069 (day 474 after transplantation) and G154 (day 336 after transplantation) showed trilineage maturation and a normal blast count. No dysplastic features were seen.

\section{Discussion}

Here, we present the first data to our knowledge evaluating MGMT(P140K) drug-resistance gene therapy in a clinically relevant large-animal model, the dog. We demonstrate that MGMT(P140K)-transduced allogeneic hematopoietic stem cells can engraft in a DLAmatched littermate, the canine equivalent of a human HLA-matched sibling, after either fully myeloablative or low-dose irradiation. We also show that these cells can be efficiently selected in vivo. We further show that MGMT(P140K) overexpression prevents the myelosuppression normally associated with coadministration of $\mathrm{O}^{6} \mathrm{BG}$ and $\mathrm{BCNU}$. Finally, we provide evidence that drug selection of genetically protected cells can be used to modulate hematopoietic donor chimerism.

The selection described here was maintained in multiple lineages for more than 12 months after drug treatment, indicating stem cell selection. After repeated drug administrations, we achieved a gene-marking level of $98 \%$

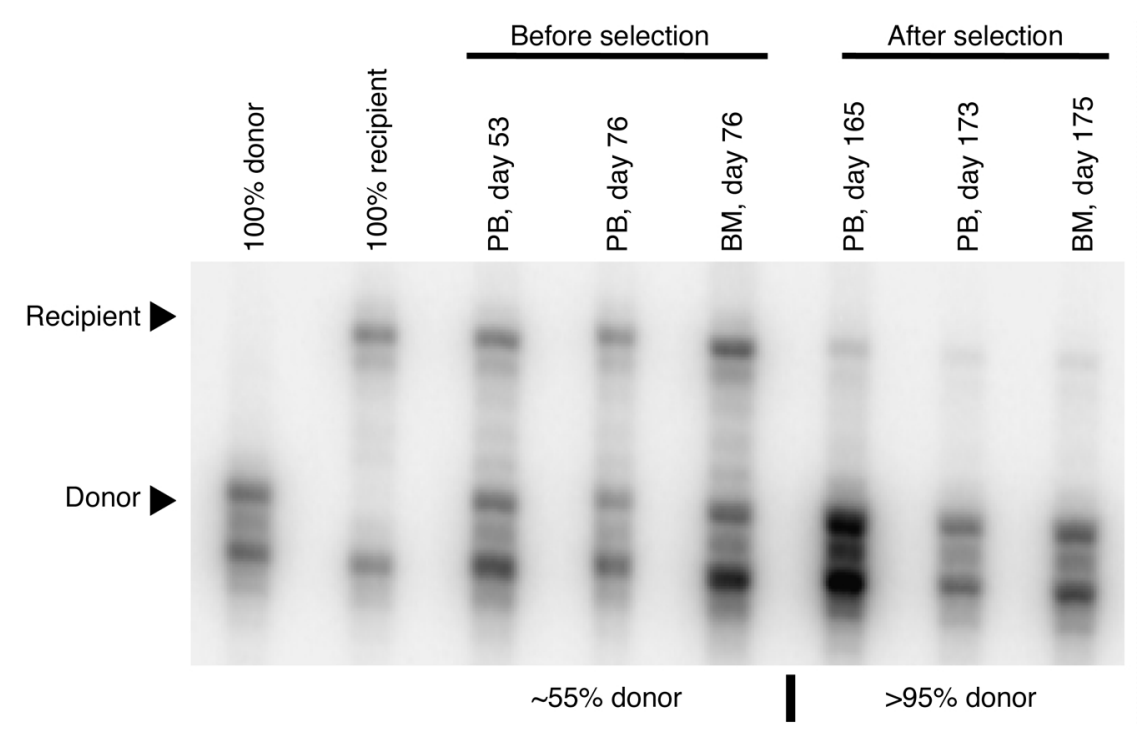

Conversion to full donor chimerism mediated by in vivo selection. VNTR analysis demonstrates an increase in donor contribution from approximately $55 \%$ to greater than $95 \%$ after in vivo selection. PB, DNA isolated from peripheral blood total nucleated cells; BM, DNA isolated from bone marrow total nucleated cells. 


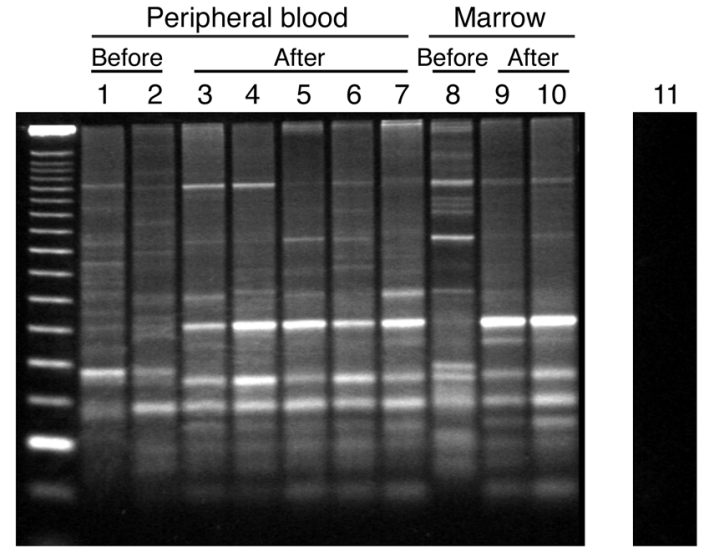

Figure 5

Protection of multiple stem cell clones. LAM-PCR-analysis of DNA derived from peripheral blood and bone marrow from dog G154 demonstrates multiple different clones that persist after in vivo selection. Before, before any selection; after, after any selection. Lanes 1-7: peripheral blood samples from day 53 (before, lane 1), day 76 (before, lane 2), day 165 (after, lane 3), day 173 (after, lane 4), day 236 (after, lane 5), day 259 (after, lane 6), day 284 (after, lane 7). Lanes 8-10: bone marrow samples from day 76 (before, lane 8), day 175 (after, lane 9), day 259 (after, lane 10). Lane 11: DNA from a normal dog used as negative control. Day 0 is the day of transplantation.

in granulocytes. We have noted transgene expression and selection in all lineages, although there are differences in the level of gene marking between different lineages. Red blood cells and platelets have lower cellular EGFP expression, which leads to an overlap of positive and negative cell populations and therefore to underestimation of the true gene marking. Expression from the lentiviral vector appeared to be somewhat brighter in platelets and less intense in red blood cells than that from the MSCV-based retroviral vector. Toxicity of the drug selection in our study was confined to the hematopoietic system and could be eliminated by a modified dose schedule using lower doses with small incremental increases instead of one large dose of BCNU in combination with $\mathrm{O}^{6} \mathrm{BG}$. No previous study in large animals or in humans to our knowledge has achieved similarly high rates of stable stem cell modification, and the in vivo gene-marking levels presented here would be curative for most genetic diseases affecting the hematopoietic system.

The myeloprotective effect of drug-resistance gene therapy has been proposed as a strategy to allow for dose intensification in the treatment of malignant tumors (32). Stem cell rescue with unmodified CD34-selected autologous cells, which has been used in the treatment of nonhematopoietic malignancy, allows for only a onetime dose escalation, a strategy that for many solid tumors has not translated into improved clinical outcome (33). In contrast, drug-resistance gene therapy would allow for repeated, tightly spaced administrations of aggressive doses of chemotherapy, bypassing the limitations imposed by myelosuppression. In this context, it is important to note that in the case of breast cancer, a more aggressive dose schedule has recently been shown to improve clinical outcome (34). Our data have significant implications for drug-resistance gene therapy in patients with solid tumors (e.g., brain tumors) for which treatment with nitrosoureas and $\mathrm{O}^{6} \mathrm{BG}$ is clinically limited by hematological toxicity (35). Incorporating a second drug-resistance gene into the vector could broaden the array of cytostatic agents useful in the context of drug-resistance gene therapy (36). Such two-gene vectors could make this strategy applicable to a wide variety of malignant diseases. The high marking level and the lack of significant toxicity observed in the present study make gene therapy using MGMT(P140K)-mediated in vivo selection of genetically corrected autologous cells also an attractive alternative to allogeneic stem cell transplantation for a number of genetic diseases.

Drug-resistance gene therapy could potentially be used to enhance allogeneic stem cell transplantation, and our data demonstrate the general feasibility of this approach. We have demonstrated that gene-modified allogeneic stem cells can stably engraft DLA-matched littermates, and mixed chimerism in one dog presented here was converted to full donor chimerism after repeated doses of the combination of $\mathrm{O}^{6} \mathrm{BG}$ and $\mathrm{BCNU}$. We were also able to show that lentivirally transduced allogeneic stem cells can engraft after a lowdose TBI conditioning regimen, with a follow-up of 10-16 weeks. Data from animals previously transplanted and published at our center suggest that allogeneic chimerism beyond 12 weeks is generally maintained in the long term (24). Several scenarios can be envisioned in which the presence of a drug-resistance gene in allogeneic stem cells would be desirable. An example would be the ability to use dose-intensive chemotherapy while protecting donor hematopoiesis in patients with persistent or recurrent disease after non-myeloablative allogeneic stem cell transplantation. Currently available treatment options in these situations, donor leukocyte infusions (DLI) in particular, are hampered by severe side effects and inconsistent success. In contrast, the administration of $\mathrm{O}^{6} \mathrm{BG}$ and $\mathrm{BCNU}$ after transplantation produced acceptable toxicity in the canine model. As an alternative to BCNU, temozolomide, a methylating agent with a favorable toxicity profile, could potentially be used in this context. Temozolomide has recently been shown to be active in the treatment of leukemia (37). Resistance to this drug may be mediated by high levels of endogenous, wild-type MGMT expression in leukemic cells. This provides an excellent rationale for the use of temozolomide in combination with $\mathrm{O}^{6} \mathrm{BG}$ to eliminate residual leukemic cells while sparing MGMT(P140K)protected donor hematopoiesis.

Drug selection could possibly be used to facilitate engraftment of allogeneic stem cells in the treatment of nonmalignant diseases. This use of drug-resistance gene therapy appears particularly attractive in the context of diseases for which vector development has been difficult (i.e., hemoglobinopathies and thalassemias). 
In vivo selection could obviate the need to include lymphocytes in the graft, thus reducing morbidity and mortality related to graft-versus-host disease.

In conclusion, our data demonstrate highly efficient in vivo selection and chemoprotection of allogeneic hematopoietic stem cells with an acceptable toxicity profile. Our findings should have direct implications for autologous gene therapy strategies for patients with genetic and malignant diseases and suggest, for the first time to our knowledge, applications for the use of genetically modified, chemoprotected stem cells in the context of allogeneic stem cell transplantation.

\section{Acknowledgments}

We would like to thank Michele Spector, the technicians in the canine facilities, and the investigators of the Program in Transplantation Biology at the Fred Hutchinson Cancer Research Center (Seattle, Washington, USA), who participated in the weekend treatments. We wish to thank Rainer Storb, Marie-Terese Little, Stacy Zellmer, and Serina Gisburne for DLA typing, Brenda Sandmaier and Peter Moore for providing canine-specific antibodies for subset analyses, Amgen Inc. for providing caninespecific growth factors, and the technicians of the hematology and pathology laboratories of the Fred Hutchinson Cancer Research Center. We would like to thank A. Dusty Miller for performing helper virus testing and Kerstin Schwarzwaelder for assisting with the LAM-PCR analysis. We thank Christopher Baum for providing the SFFV promoter. We would also like to thank Robert Hackman for morphological analysis of bone marrow smears. This work was supported by NIH grants HL36444, HL074162, HL54881, DK47754, DK02753, and DK56465.

1. Cavazzana-Calvo, M., et al. 2000. Gene therapy of human severe combined immunodeficiency (SCID)-X1 disease. Science. 288:669-672.

2. Hacein-Bey-Abina, S., et al. 2002. Sustained correction of X-linked severe combined immunodeficiency by ex vivo gene therapy. N. Engl. J. Med. 346:1185-1193.

3. Aiuti, A., et al. 2002. Correction of ADA-SCID by stem cell gene therapy combined with nonmyeloablative conditioning. Science. 296:2410-2413.

4. Williams, D.A., and Smith, F.O. 2000. Progress in the use of gene transfer methods to treat genetic blood diseases. Hum. Gene Ther. 11:2059-2066.

5. Kurre, P., and Kiem, H.P. 2000. Progress towards hematopoietic stem cell gene therapy. Curr. Opin. Mol. Ther. 2:400-411.

6. Noguchi, P. 2003. Risks and benefits of gene therapy. N. Engl. J. Med. 348:193-194.

7. Hanenberg, H., et al. 1996. Colocalization of retrovirus and target cells on specific fibronectin fragments increases genetic transduction of mammalian cells. Nat. Med. 2:876-882.

8. Kiem, H.P., et al. 1998. Improved gene transfer into baboon marrow repopulating cells using recombinant human fibronectin fragment $\mathrm{CH}-296$ in combination with interleukin-6, stem cell factor, FLT-3 ligand, and megakaryocyte growth and development factor. Blood. 92:1878-1886.

9. Tisdale, J.F., et al. 1998. Ex vivo expansion of genetically marked rhesus peripheral blood progenitor cells results in diminished long-term repopulating ability. Blood. 92:1131-1141.

10. Goerner, M., et al. 1999. The use of granulocyte colony-stimulating factor during retroviral transduction on fibronectin fragment $\mathrm{CH}-296$ enhances gene transfer into hematopoietic repopulating cells in dogs. Blood. 94:2287-2292

11. Kiem, H.P., et al. 1999. Improved gene transfer into canine hematopoietic repopulating cells using CD34-enriched marrow cells in combination with a gibbon ape leukemia virus-pseudotype retroviral vector. Gene Ther. 6:966-972.

12. Kelly, P.F., Vandergriff, J., Nathwani, A., Nienhuis, A.W., and Vanin, E.F. 2000. Highly efficient gene transfer into cord blood nonobese diabetic/severe combined immunodeficiency repopulating cells by oncoretroviral vector particles pseudotyped with the feline endogenous retrovirus (RD114) envelope protein. Blood. 96:1206-1214.

13. Goerner, M., et al. 2001. Sustained multilineage gene persistence and expression in dogs transplanted with CD $34^{+}$marrow cells transduced by RD114-pseudotype oncoretrovirus vectors. Blood. 98:2065-2070.

14. Horn, P.A., Topp, M.S., Morris, J.C., Riddell, S.R., and Kiem, H.P. 2002. Highly efficient gene transfer into baboon marrow repopulating cells using GALV-pseudotype oncoretroviral vectors produced by human packaging cells. Blood. 100:3960-3967.

15. Huhn, R.D., et al. 1999. Retroviral marking and transplantation of rhesus hematopoietic cells by nonmyeloablative conditioning. Hum. Gene Ther. 10:1783-1790.

16. Neff, T., and Blau, C.A. 2001. Pharmacologically regulated cell therapy. Blood. 97:2535-2540.

17. Neff, T., et al. 2002. Pharmacologically regulated in vivo selection in a large animal. Blood. 100:2026-2031.

18. Sorrentino, B.P. 2002. Gene therapy to protect haematopoietic cells from cytotoxic cancer drugs. Nat. Rev. Cancer. 2:431-441.

19. Ragg, S., et al. 2000. Direct reversal of DNA damage by mutant methyltransferase protein protects mice against dose-intensified chemotherapy and leads to in vivo selection of hematopoietic stem cells. Cancer Res. 60:5187-5195.

20. Davis, B.M., Koc, O.N., and Gerson, S.L. 2000. Limiting numbers of G156A $\mathrm{O}^{6}$-methylguanine-DNA methyltransferase-transduced marrow progenitors repopulate nonmyeloablated mice after drug selection. Blood. 95:3078-3084.

21. Persons, D.A., et al. 2003. Successful treatment of murine $\beta$-thalassemia using in vivo selection of genetically modified, drug-resistant hematopoietic stem cells. Blood. 102:506-513.

22. Maris, M., and Storb, R. 2002. Outpatient allografting in hematologic malignancies and nonmalignant disorders: applying lessons learned in the canine model to humans. In Non-myeloablative allogeneic transplantation. A. Bashey and E.D. Ball, editors. Kluwer Academic Publishers. Boston, Massachusetts, USA. 149-175.

23. Wagner, J.L., Works, J.D., and Storb, R. 1998. DLA-DRB1 and DLA-DQB1 histocompatibility typing by PCR-SSCP and sequencing. Tissue Antigens. 52:397-401.

24. Storb, R., et al. 1997. Stable mixed hematopoietic chimerism in DLA-identical littermate dogs given sublethal total body irradiation before and pharmacological immunosuppression after marrow transplantation. Blood. 89:3048-3054.

25. McSweeney, P.A., et al. 1998. Characterization of monoclonal antibodies that recognize canine CD34. Blood. 91:1977-1986.

26. Williams, D.A., et al. 2000. Dominant negative mutation of the hematopoietic-specific Rho GTPase, Rac2, is associated with a human phagocyte immunodeficiency. Blood. 96:1646-1654.

27. Follenzi, A., Ailles, L.E., Bakovic, S., Geuna, M., and Naldini, L. 2000. Gene transfer by lentiviral vectors is limited by nuclear translocation and rescued by HIV-1 pol sequences. Nat. Genet. 25:217-222.

28. Horn, P.A., et al. 2002. Lentivirus-mediated gene transfer into hematopoietic repopulating cells in baboons. Gene Ther. 9:1464-1471.

29. Georges, G.E., et al. 2000. Adoptive immunotherapy in canine mixed chimeras after nonmyeloablative hematopoietic cell transplantation. Blood. 95:3262-3269.

30. Schmidt, M., et al. 2003. Clonality analysis after retroviral-mediated gene transfer to $\mathrm{CD}_{34}{ }^{+}$cells from the cord blood of ADA-deficient SCID neonates. Nat. Med. 9:463-468.

31. Schmidt, M., et al. 2002. Polyclonal long-term repopulating stem cell clones in a primate model. Blood. 100:2737-2743.

32. Abonour, R., et al. 2000. Efficient retrovirus-mediated transfer of the multidrug resistance 1 gene into autologous human long-term repopulating hematopoietic stem cells. Nat. Med. 6:652-658.

33. Stadtmauer, E.A., et al. 2000. Conventional-dose chemotherapy compared with high-dose chemotherapy plus autologous hematopoietic stem-cell transplantation for metastatic breast cancer. Philadelphia Bone Marrow Transplant Group. N. Engl. J. Med. 342:1069-1076.

34. Citron, M.L., et al. 2003. Randomized trial of dose-dense versus conventionally scheduled and sequential versus concurrent combination chemotherapy as postoperative adjuvant treatment of node-positive primary breast cancer: first report of Intergroup Trial C9741/Cancer and Leukemia Group B Trial 9741. J. Clin. Oncol. 21:1431-1439.

35. Quinn, J.A., et al. 2002. Phase II trial of carmustine plus $\mathrm{O}^{6}$-benzylguanine for patients with nitrosourea-resistant recurrent or progressive malignant glioma. J. Clin. Oncol. 20:2277-2283.

36. Rappa, G., Lorico, A., Hildinger, M., Fodstad, O., and Baum, C. 2001. Novel bicistronic retroviral vector expressing gamma-glutamylcysteine synthetase and the multidrug resistance protein 1 (MRP1) protects cells from MRP1-effluxed drugs and alkylating agents. Hum. Gene Ther. 12:1785-1796

37. Seiter, K., et al. 2002. Phase I study of temozolomide in relapsed/refractory acute leukemia. J. Clin. Oncol. 20:3249-3253. 